\title{
LOS PELTEPHILIDAE (MAMMALIA, XENARTHRA) DE LA FORMACIÓN COLLÓN CURÁ (COLLONCURENSE, MIOCENO MEDIO), ARGENTINA
}

\author{
LAUREANO RAÚL GONZÁLEZ-RUIZ \\ Laboratorio de Investigaciones en Evolución y Biodiversidad, Universidad Nacional de La Patagonia San Juan Bosco, \\ CONICET, Ruta Nacional 259, km 16.5, 9200, Esquel, Argentina. gonzalezlaureano@yahoo.com.ar

\section{FLÁVIO GÓIS} \\ Centro de Investigaciones Científicas y de Transferencia de Tecnología a la Producción, CONICET, \\ Matteri y España, 3105, Diamante, Argentina.fgois@cicyttp.org.ar \\ MARTIN RICARDO CIANCIO \& GUSTAVO JUAN SCILLATO-YANÉ \\ Departamento Científico Paleontología Vertebrados, Museo de La Plata, Paseo del Bosque, s/n, 1900, \\ La Plata, Argentina, CONICET, mciancio@fcnym.unlp.edu.ar,scillato@fcnym.unlp.edu.ar
}

\begin{abstract}
THE PELTEPHILIDAE (MAMMALIA, XENARTHRA) FROM THE COLLÓN CURÁ FORMATION (COLLONCURAN, MIDDLE MIOCENE), ARGENTINA. The Peltephilidae (early Eocene-late Miocene) are a group of extinct armadillos exclusive from South America, known for having horn-like osteoderms on the nasals and maxillaries. This paper discusses a nomenclatorial proposal for anatomical description of these cingulate osteoderms and describes for the first time specimens of Peltephilidae (Xenarthra, Cingulata) from the Collón Curá Formation (Colloncuran, middle Miocene) of Patagonia (Argentina). The major change in the nomenclature of the osteoderms in Peltephilidae here proposed is to replace the term "central or main figure" by "central longitudinal elevation". Finally, by analogy with the living species of Dasypodidae and the extinct Pampatheriidae, some considerations are presented about the relationships between the osteoderm and the overlying epidermal scale in Peltephilidae. The new specimens are assigned to Peltephilidae gen. et sp. indet., Peltephilus pumilus Ameghino and P. nanus Ameghino. The latter two species represent their youngest record and indicate a closer taxonomic affinity of the Colloncuran age with the previous Santacrucian, instead of the subsequent Laventan and Mayoan.
\end{abstract}

Key words: Peltephilidae, Xenarthra, middle Miocene, Colloncuran, Patagonia, Argentina.

RESUMO - Os Peltephilidae (Eoceno inferior-Mioceno superior) são um grupo de cingulados extintos exclusivos da América do Sul, conhecidos por apresentar alguns osteodermos cefálicos diferenciados num par de cornos pré-orbitários. Neste trabalho se discute uma proposta nomenclatural para a descrição anatômica dos osteodermos desses cingulados e se descrevem pela primeira vez restos de Peltephilidae (Xenarthra, Cingulata) para a Formação Collón Curá (Colloncurense, Mioceno médio) da Patagônia (Argentina). A principal mudança na nomenclatura dos osteodermos dos Peltephilidae aqui proposta, é a substituição do termo "figura central ou principal" por "elevação central longitudinal". Finalmente, por analogia com as espécies de Dasypodidae atuais e Pampatheriidae são apresentadas algumas considerações sobre a relação entre o osteodermo e a escama epidérmica sobrejacente nos Peltephilidae. Os novos espécimens são atribuídos a Peltephilidae gen. et sp. indet., Peltephilus pumilus Ameghino e P. nanus Ameghino. Os últimos dois registros são os mais recentes para ambas espécies e indicam uma maior afinidade taxonômica da idade Colloncurense à precedente Santacruzense, do que com as posteriores Laventense e Maioense.

Palavras-chaves: Peltephilidae, Xenarthra, Mioceno médio, Colloncurense, Patagônia, Argentina.

\section{INTRODUCCIÓN}

Los Peltephilidae son un grupo de armadillos extinctos exclusivos de América del Sur. Están representados por cinco géneros y 13 especies (Scillato-Yané, 1980; Croft et al., 2007; González Ruiz et al., 2012). Además, tres probables nuevas especies han sido citadas en la literatura (de Barrio et al., 1984; Croft et al., 2009; Bostelmann et al., 2012).
Su biocrón se extiende desde el Eoceno temprano ( $c a .58 .5-55$ Ma. Riochiquense) hasta el Mioceno tardío ( $c a$. 10.0?-9.0 Ma. Chasiquense) (Scillato-Yané, 1986; Flynn \& Swisher, 1995; Tejedor et al., 2009; González Ruiz et al., 2012).

Los análisis filogenéticos indican que Peltephilus Ameghino, 1887 (genotipo de la familia Peltephilidae) se ubica en una posición basal dentro del clado de los Cingulata; según Abrantes \& Bergqvist (2006) es el taxón hermano de 
todos los Dasypodidae (sensu McKenna \& Bell, 1997), según Gaudin \& Wible (2006), Billet et al. (2011) y Ciancio (2010) Peltephilus es el grupo hermano de todos los demás cingulados. Para Ciancio (2010), Peltephilus constituye un clado junto con Machlydotherium Ameghino, 1902. Esta posición basal de Peltephilus también es apoyada por los resultados del estudio paleohistológico de sus osteodermos (Wolf, 2007).

$\mathrm{Al}$ igual que el resto de los cingulados, los Peltephilidae poseen una coraza de osteodermos dérmicos cubiertos por escamas epidérmicas, conformada por un escudo cefálico y una coraza dorsal (Ameghino, 1894; Lahille, 1895; Scott, 1903; Cooper, 1930; Grassé, 1955; Edmund, 1985; Engelmann, 1985; Wolf, 2007; Krmpotic et al., 2009). La existencia de un estuche caudal y un escudo escapular (conformando parte de la coraza dorsal) aún se discute, dado que no se conocen corazas completas para los Peltephilidae. Los especímenes conocidos permiten reconocer en la coraza dorsal una región anterior de osteodermos móviles (imbricados) y una posterior o escudo pélvico de osteodermos no fusionados (no imbricados) (Ameghino, 1889, 1894, 1895; Scott, 1903; Scillato-Yané, 1982). En el escudo cefálico se verifica uno de los rasgos más notorios de los peltefílidos, la presencia de uno o dos pares de osteodermos sobre los nasales y maxilares formando elevaciones a modo de "cuernos" (Ameghino, 1894; Scott, 1903; Bordas, 1936).

Los osteodermos de los Peltephilidae tienen una morfología propia que permiten diferenciarlos fácilmente de los demás cingulados. Uno de los rasgos más evidentes es la ausencia de figuras delimitadas por surcos en la superficie expuesta del osteodermo (i.e. central y periféricas) por lo que la nomenclatura usual utilizada para la descripción de los osteodermos de la mayoría de los Cingulata (i.e. Glyptodontidae y Dasypodidae) (Ameghino, 1889; Lahille, 1895) resulta inadecuada. Recientemente, Góis et al. (2013) han propuesto una nueva nomenclatura para describir de manera más precisa la superficie expuesta de los osteodermos de los Pampatheriidae y sugieren que ésta también podría ser aplicable a los osteodermos de otros Cingulata carentes de figuras, como sucede en los Peltephilidae.

El conocimiento de los peltefílidos del Mioceno medio (post-santacrucenses), en particular los del Colloncurense (ca. 15.5-14 Ma., Mioceno medio; Flynn \& Swisher, 1995) es muy pobre y sólo se los ha mencionado a nivel familiar para la Formación Collón Curá de la Provincia de Río Negro (Casamiquela, 1963; Ganduglia, 1977; Coira, 1979; Pascual et al., 1984; Pardiñas \& Reguero, 1987). Las citas corresponden a hallazgos de restos aislados en las localidades Cantera 27 de Setiembre, Cantera Lif Mahuida y Cerro Castillo (Casamiquela, 1963; Ganduglia, 1977; Pardiñas \& Reguero, 1987) (Figura 1). Estos especímenes nunca han sido descriptos formalmente, ni figurados o ingresados formalmente a ningún repositorio.

En suma, en este trabajo se describe por primera vez restos de Peltephilidae (Xenarthra, Cingulata) para la Formación

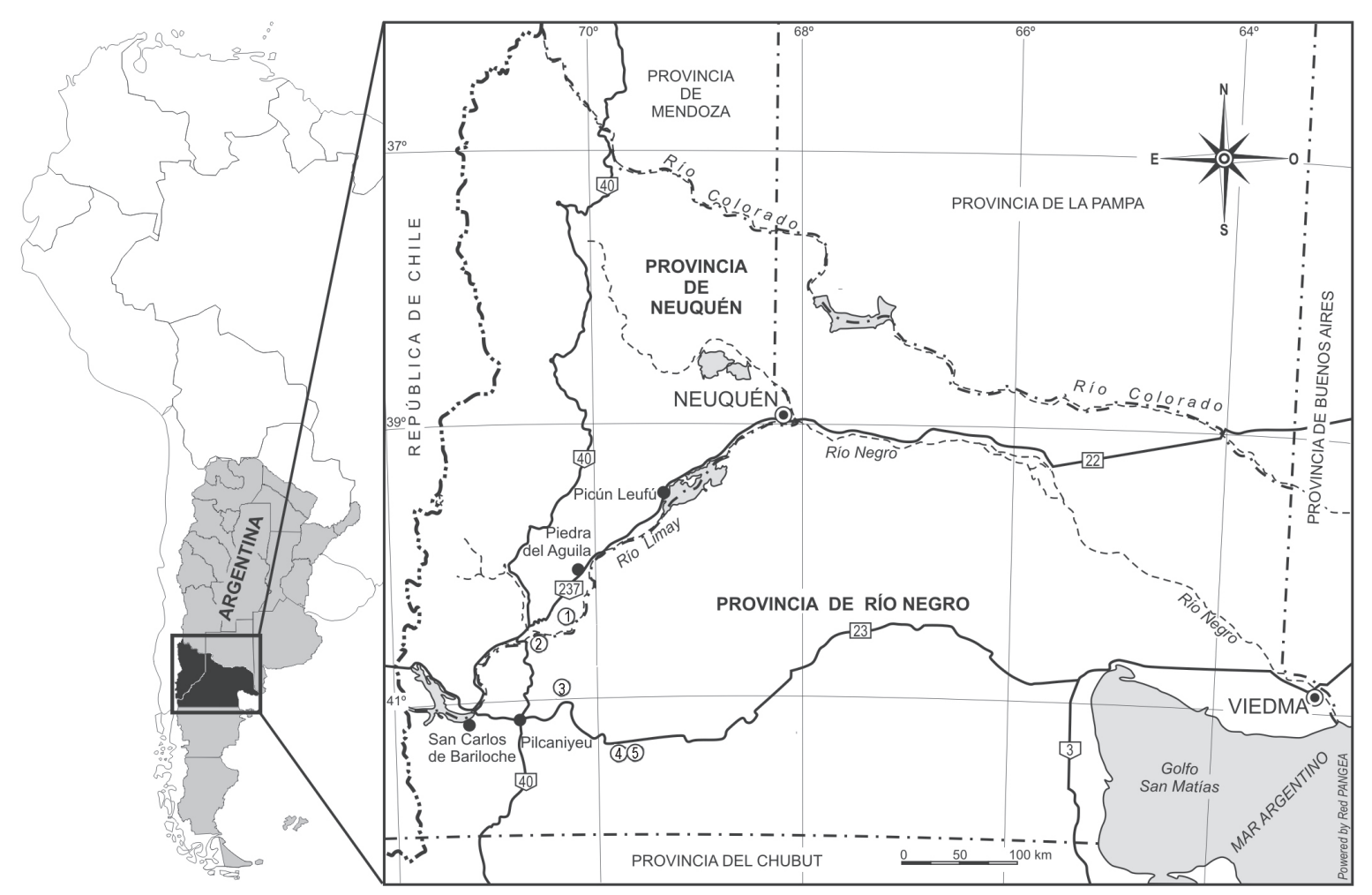

Figura 1. Mapa de localización del área de estudio y localidades fosilíferas citadas en el texto. 1, Cañadón del Tordillo; 2, Cerro Castillo; 3, Estancia Criado; 4, Cantera 27 de Setiembre; 5, Cantera Lif Mahuida.

Figure 1. Location map of the study area and fossiliferous localities mentioned in the text. 1, Cañadón del Tordillo; 2, Cerro Castillo; 3, Estancia Criado; 4, Cantera 27 de Setiembre; 5, Cantera Lif Mahuida. 
Collón Curá (Colloncurense, Mioceno medio) de Patagonia (Argentina) y se propone el empleo de una nueva nomenclatura para describir los osteodermos de los Peltephilidae, basándose en la nomenclatura recientemente propuesta por Góis et al. (2013) para los Pampatheriidae. Estos registros consisten en osteodermos desarticulados de la coraza dorsal recolectados en afloramientos de la Formación Collón Curá de las localidades Cañadón del Tordillo (Provincia del Neuquén) y Estancia Criado (Provincia de Río Negro; Figura 1).

\section{CONTEXTO ESTRATIGRÁFICO Y EDAD DE LA FORMACIÓN COLLÓN CURÁ}

Los afloramientos de las provincias de Río Negro y Neuquén pertenecientes a la Formación Collón Curá, fueron originalmente dados a conocer por Roth (1899) a fines del siglo 19 quién los estudiara en las barrancas del río Collón Curá (Provincia del Neuquén). Numerosos trabajos geológicos se han realizado desde entonces en la región (Galli, 1969; Rabassa, 1975, 1978; Nullo, 1978; Coira, 1979; Bondesio et al., 1980; Leanza et al., 1997; Cucchi et al., 1998), aunque es el trabajo de Vucetich et al. (1993) donde se presenta una descripción estratigráfica detallada de la Formación Collón Curá en la localidad Cañadón del Tordillo (Provincia del Neuquén).

Vucetich et al. (1993) sugieren denominar faunas colloncurenses a aquellas procedentes de la Formación Collón Curá y que están en asociación directa con la Ignimbrita Pilcaniyeu (unidad estratigráfica guía) (Mazzoni \& Stura, 1990; Mazzoni, 1993). Si bien se encontraron fósiles por debajo y por encima de dicha ignimbrita, los estudios sedimentológicos indican una depositación relativamente rápida, por lo que los fósiles de ambos horizontes se consideran colloncurenses.

Tradicionalmente, las faunas Friasense, Colloncurense y Mayoense conformaban la Edad-Mamífero "Friasense" (Kraglievich, 1930; Pascual \& Odreman, 1973). Con el reconocimiento de nuevas faunas y el estudio de las ya conocidas, este esquema de Edades-Mamífero Sudamericanas para el Mioceno medio quedó conformado por cuatro edades consecutivas (Friasense s.s., Colloncurense, Laventense y Mayoense) (Flynn \& Swisher, 1995; Madden et al., 1997; Croft et al., 2007).

En el sentido estrictamente cronológico, se han publicado diversas dataciones ${ }^{40} \mathrm{~K} /{ }^{39} \mathrm{Ar}$ para la Formación Collón Curá que van desde los 10.7 Ma. (Cazau et al., 1989) a los 16.1 \pm 2.6 Ma. (Mazzoni \& Benvenuto, 1990) (ver también Rabassa, 1975; Marshall et al., 1977). La más moderna y única datación ${ }^{40} \mathrm{Ar} /{ }^{39} \mathrm{Ar}$ de la Ignimbrita Pilcaniyeu arrojó una edad de 15.7 Ma. (Flynn \& Swisher, 1995; Madden et al., 1997). Esta última edad ubica a la Formación Collón Curá y a los sedimentos portadores de la fauna colloncurense en el Mioceno medio.

\section{MATERIAL Y MÉTODOS}

La macrosistemática utilizada es aquélla propuesta y compendiada por Pascual et al. (1990) y modificada por
McKenna \& Bell (1997), y el esquema geocronológico empleado es el de Flynn \& Swisher (1995) modificado por Croft et al. (2009).

Todos los valores se expresan en milímetros $(\mathrm{mm})$ y se midieron directamente en los especímenes con un calibre digital con un rango de error de $0,5 \mathrm{~mm}$. El área total de cada osteodermo (ATO) y el área del foramen derecho (AFD) se calcularon con el programa ImageJ 1.32 (http:// rsb.info.nih.gov).

\section{Nomenclatura de los osteodermos de los Peltephilidae}

Al intentar reconstruir la historia evolutiva de los Cingulata se ha encontrado con el inconveniente de que muchas de las especies fósiles de cingulados, incluidos algunas de los peltefílidos se conocen básicamente por osteodermos desarticulados (Ameghino, 1887, 1889, 1894, 1898, 1902; Simpson, 1948; Scillato-Yané, 1980, 1982; Oliveira \& Bergqvist, 1998; Ciancio \& Carlini, 2008a; Krmpotic et al., 2009; Carlini et al., 2010).

A pesar de que el uso exclusivo de los caracteres de los osteodermos en la taxonomía de los cingulados puede resultar en el reconocimiento de taxones artificiales (Simpson, 1948; Hoffstetter, 1958; Perea, 2005), también es cierto que cuando es posible comparar osteodermos de regiones homólogas de la coraza y analizar la variación morfológica de las especies vivientes de Dasypodidae, se pone en evidencia su alto valor diagnóstico (González \& Scillato-Yané, 2008; Carlini et al., 2010; Ciancio, 2010; González, 2010a).

Sobre la morfología externa de los osteodermos, así como sobre la disposición de las distintas secciones de la coraza, se han construido la mayoría de los esquemas sistemáticos de los Cingulata, aunque no siempre bajo la misma nomenclatura (Ciancio, 2010). Tradicionalmente la nomenclatura utilizada para describir los osteodermos de los Peltephilidae han sido los mismos empleados para los osteodermos de los Dasypodidae y Glyptodontidae (Ameghino, 1887, 1889; Lahille, 1895; Scillato-Yané, 1982; Vizcaíno et al., 2003; Hill, 2006; Croft et al., 2007; Krmpotic et al., 2009; Oliveira \& Pereira, 2009; Fernicola \& Porpino, 2012).

Sin embargo, los osteodermos de la coraza dorsal de los Peltephilidae poseen una morfología muy particular. Estos se caracterizan en general por ser rectangulares o cuadrangulares, proporcionalmente delgados, anchos, con una superficie rugosa, sin figuras diferenciadas y presentan típicamente de dos (excepcionalmente uno) a cuatro forámenes de gran tamaño en su superficie externa. Los osteodermos móviles (imbricados) poseen la región articular anterior corta y ancha. Los osteodermos fijos (no imbricados) del escudo pélvico son más cortos y anchos que los móviles, sin región articular anterior y, a diferencia del de otros Dasypodidae, estarían simplemente yuxtapuestos y unidos de manera laxa sin suturas (Ameghino, 1887, 1889, 1895; Lydekker, 1894; Scott, 1903; Scillato-Yané, 1982).

Según observaciones los osteodermos sin región articular anterior desarrollada de los autores, (fijos o no imbricados) pueden ser pentagonales o hexagonales con los vértices redondeados o casi circulares, mientras los que 
poseen la región articular anterior desarrollada (móviles o imbricados) son rectangulares o cuadrangulares. Dado que no se han recuperado corazas articuladas de Peltephilidae existe la posibilidad que estos osteodermos sin región articular desarrollada pertenezcan a la región más anterior y/o más posterior de la coraza dorsal (Ameghino, 1895) o exclusivamente a la región más posterior de la coraza (Scott, 1903; Scillato-Yané, 1982).

Una de las características más notable, es la ausencia de figuras en la superficie externa, lo que también puede observarse en otros Cingulata (i.e. Pampatheriidae, ver Góis et al. 2013). Esta carencia implica que no pueden ser descriptos adecuadamente con la terminología estándar aplicada para la ornamentación de los Dasypodidae o de los Glyptodontidae. En este contexto resulta útil la nomenclatura propuesta por Góis et al. (2013) para la descripción de los Pampatheriidae, y que aquí se propone usarla para los Peltephilidae. Esta nomenclatura implica los siguientes descriptores (Figura 2): (i) margen lateral o márgenes laterales, donde pueden ubicarse forámenes; (ii) elevación marginal que delimita la superficie externa; (iii) depresión longitudinal, ubicada entre los márgenes laterales y la elevación central longitudinal; (iv) elevación central longitudinal, la cual se ubica en el centro de la superficie externa, entre las depresiones longitudinales y al igual que estas tiene una disposición anteroposterior.
Abreviaturas institucionales. MACN-A, Colección Nacional Ameghino, Museo Argentino de Ciencias Naturales "Bernardino Rivadavia", Buenos Aires, Argentina; MLP, División Paleontología Vertebrados, Facultad de Ciencias Naturales y Museo, Universidad Nacional de La Plata, La Plata, Argentina.

\section{SISTEMÁTICA PALEONTOLÓGICA}

Superorden XENARTHRA Cope, 1889

Orden CINGULATA Illiger, 1811

Superfamilia DASYPODOIDEA Gray, 1821

Familia PELTEPHILIDAE Ameghino, 1894

Peltephilidae gen. et sp. indet.

(Figura 3A)

Material referido. MLP 74-II-1-23, un fragmento de osteodermo del escudo cefálico.

Procedencia geográfica y estratigráfica. Estancia Criado, Pilcaniyeu Viejo (Provincia de Río Negro) (Figura 1), Formación Collón Curá, Mioceno medio (Bondesio et al., 1980).

Descripción y comentarios. El espécimen corresponde a un fragmento de osteodermo del escudo cefálico; posee numerosos forámenes de pequeño tamaño en su superficie y una elevación marginal delimitándola. Por fuera de esta última se ubica una hilera de forámenes.

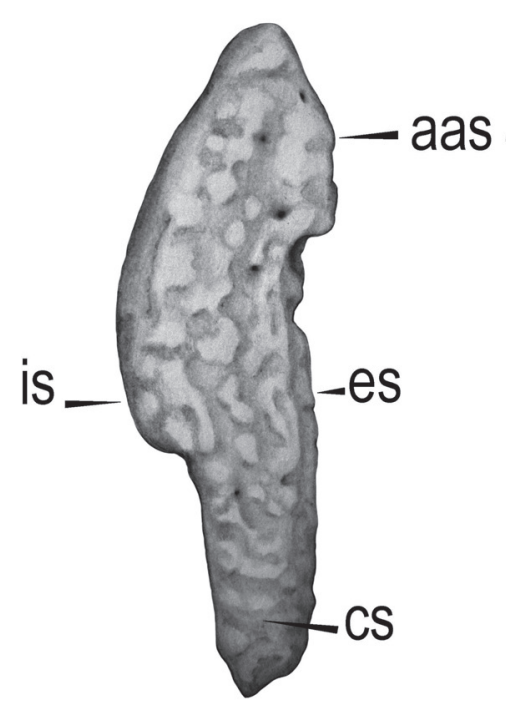

A

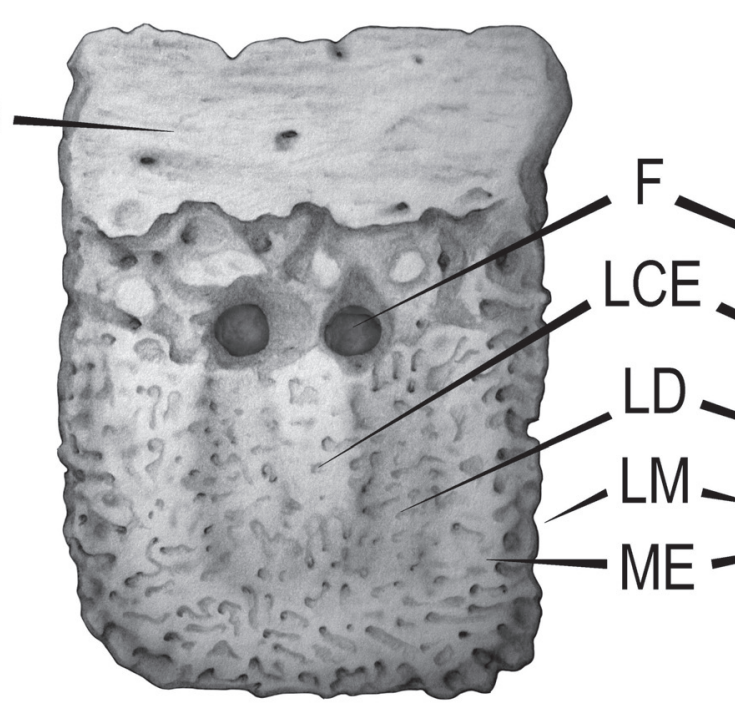

B 
El escudo cefálico de los Peltephilidae consiste en 1921 osteodermos grandes y gruesos ordenados en un patrón definido. En la línea media se ubican tres osteodermos simétricos impares, de los cuales el más posterior es de forma hexagonal algo irregular y es el de mayor tamaño del escudo cefálico (y de toda la coraza conocida). Este osteodermo varía en su forma y proporciones en las diferentes especies. El segundo osteodermo de esta línea media es más largo y angosto, mientras que el más anterior es mucho más pequeño. A cada lado de la línea media hay siete osteodermos de diferentes tamaños y formas poligonales, excepto el de posición postorbital que termina en una punta roma. Todos los osteodermos del escudo cefálico poseen una superficie muy rugosa y gruesamente esculpida, dichos dibujos parecerían constantes para cada especie (Scott, 1903).

Los escudos cefálicos más completos conocidos de Peltephilidae pertenecen a Peltephilus strepens, Ameghino, 1887, P. ferox Ameghino, 1891 y P. giganteus Ameghino, 1894 (Ameghino, 1889; Moreno \& Mercerat, 1891; Ameghino, 1894; Scott, 1903) pero en el estado actual del conocimiento solo es posible identificar las especies antedichas cuando conservan el osteodermo cefálico central (Ameghino, 1898, 1920; Scott, 1903; González, 2010a). En el caso del MLP 74-II-1-23 la porción conservada corresponde a un fragmento osteodermo de posición lateral y no posee caracteres para una identificación más precisa.

\section{Peltephilus Ameghino, 1887}

Especie tipo. Peltephilus strepens Ameghino, 1887.

Peltephilus pumilus Ameghino, 1887

(Figuras 3B-C; 4A-L)

Material referido. MLP 91-IV-1-62, un osteodermo móvil de la coraza dorsal (Figuras 3B-C).

Procedencia geográfica y estratigráfica. Cañadón del Tordillo (Provincia del Neuquén) (Figura 1), Formación Collón Curá, Mioceno medio (Pardiñas, 1991; Vucetich et al., 1993). Colector: U. Pardiñas.

Descripción y comentarios. El material tipo de esta especie aún no ha podido ubicarse con certeza, aunque hay tres lotes de osteodermos que coinciden con las descripciones originales de Ameghino $(1887,1889)$ que podrían incluir parte o toda la serie tipo original (MLP 67-XI-13-1 a 3, MLP 67-VIII-121c, MACN A 866-870) (González, 2010a) (Figuras 4A-L).

De acuerdo a lo observado en estos restos los osteodermos de Peltephilus pumilus se caracterizan por ser de un tamaño menor (ca. 50\%) que los de las demás especies conocidas de Peltephilidae (excepto P. nanus Ameghino, 1898). Los osteodermos móviles miden 12.1-14.1 de largo x 7.7-12.1 de ancho y los fijos miden 10.9-12.5 de largo x 8.7-9.6 de ancho. Generalmente, los osteodermos presentan la elevación central longitudinal angosta y elevada y el par de forámenes anteriores más desarrollados que los de $P$. strepens (Ameghino, 1887, 1889; Kramarz et al., 2010; González, 2010a). En la región anterior del osteodermo por encima de los grandes forámenes se desarrollan una serie tubérculos ubicados aproximadamente en una hilera transversal (Kramarz et al., 2010).

La relación promedio entre el área del foramen derecho (AFD) y el área total del osteodermo (ATO) para los osteodermos de Peltephilus pumilus estudiados es menor que para $P$. nanus. Es decir que los forámenes de $P$. pumilus en promedio son de menor tamaño en relación al tamaño del osteodermo que los de P. nanus (ver Tabla 1).

El resto que aquí se describe pertenece a un osteodermo de las bandas móviles, sobre su cara externa presenta la rugosidad y los dos forámenes anteriores característicos de la mayoría de las especies de Peltephilus. Mide 10.3 de largo x 9.5 de ancho, tamaño que lo ubica en el rango de los osteodermos móviles de $P$. pumilus siendo un poco mayor que los de $P$. nanus. Este osteodermo no posee elevaciones longitudinales desarrolladas y su superficie es plana, pero presenta una hilera de cuatro tubérculos anteriores por delante de los dos forámenes que permiten asignarlo a $P$. pumilus.

Tabla 1. Medidas de osteodermos (mm) de Peltephilus pumilus y P. nanus. Abreviaturas: AFD, area foramen derecho; ATO, area total osteodermo.

Table 1. Measurements of osteoderms ( $\mathrm{mm}$ ) of Peltephilus pumilus and $P$. nanus. Abbreviations: AFD, right foramen area; ATO, total area osteoderm.

\begin{tabular}{|c|c|c|c|}
\hline Osteodermos & AFD & ATO & $\mathrm{AFD} / \mathrm{ATO}$ \\
\hline \multicolumn{4}{|l|}{ P. pumilus } \\
\hline \multicolumn{4}{|l|}{ MACN A 866-870 } \\
\hline Móvil 1 & 5,6 & 132,4 & 0,0423 \\
\hline Móvil 2 & 2,4 & 82,7 & 0,0290 \\
\hline Móvil 3 & 1,6 & 95,4 & 0,0168 \\
\hline Fijo & 6,2 & 91,8 & 0,0675 \\
\hline \multicolumn{4}{|l|}{ MLP 67-XI-13-1a3 } \\
\hline Móvil 1 & 5,3 & 121,9 & 0,0435 \\
\hline Móvil 2 & 3,7 & 126,1 & 0,0293 \\
\hline Fijo & 2,7 & 86,9 & 0,0311 \\
\hline \multicolumn{4}{|l|}{ MLP 67-VIII-12-1c } \\
\hline Móvil 1 & 4,6 & 122,9 & 0,0423 \\
\hline Móvil 2 & 4,1 & 88,8 & 0,0462 \\
\hline \multicolumn{4}{|l|}{ MLP 91-IV-1-62 } \\
\hline Móvil & 5,2 & 96,5 & 0,0539 \\
\hline Promedio AFD/ATO & & & 0,0460 \\
\hline \multicolumn{4}{|l|}{ P. nanus } \\
\hline \multicolumn{4}{|l|}{ MACN A 7958-7959 } \\
\hline Móvil & 2,6 & 43,1 & 0,0603 \\
\hline Fijo & 3,7 & 54,3 & 0,0681 \\
\hline \multicolumn{4}{|l|}{ MLP 91-IV-1-35 } \\
\hline Móvil 1 & 4,5 & 71,8 & 0,0627 \\
\hline Móvil 2 & 3,7 & 64,5 & 0,0574 \\
\hline \multicolumn{4}{|l|}{ MLP 91-IV1-134 } \\
\hline Móvil & 3,4 & 65,7 & 0,0518 \\
\hline Fijo 1 & 4,1 & 52,2 & 0,0785 \\
\hline Fijo 2 & 4,2 & 57,7 & 0,0728 \\
\hline Promedio AFD/ATO & & & 0,0644 \\
\hline
\end{tabular}


Peltephilus nanus Ameghino, 1898

(Figuras 3D-J; 4M-N)

Materiales referidos. MLP 91-IV-1-35, dos osteodermos completos y dos fragmentos de osteodermos móviles de la coraza dorsal (Figuras 3D-G); MLP 91-IV-1-134, un osteodermo móvil y dos osteodermos fijos de la coraza dorsal (Figuras 3H-J).

Procedencia geográfica y estratigráfica. Cañadón del Tordillo (Provincia del Neuquén) (Figura 1), Formación Collón Curá, Mioceno medio (Pardiñas, 1991; Vucetich et al., 1993). Colector: U. Pardiñas.
Descripción y comentarios. El material tipo de Peltephilus nanus consiste en un fémurizquierdo, un osteodermo móvilyuno fijo de la coraza dorsal (MACNA 7958-7959) (Figuras 4M-N). En este caso los osteodermos son de un tamaño menor que los de P. pumilus (ca. $50 \%$ ), el osteodermo móvil mide 8.0 de largo x 5.7 de ancho mientras que el fijo mide 8.6 de largo x 6.1 de ancho. Presentan la superficie rugosa y los dos forámenes típicos de Peltephilus en la región anterior, y las elevaciones longitudinales están muy poco indicadas (Ameghino, 1898; Croft et al., 2007; González, 2010a,b; González Ruiz et al., 2012). Inversamente a P. pumilus, la relación promedio AFD/ATO en los osteodermos
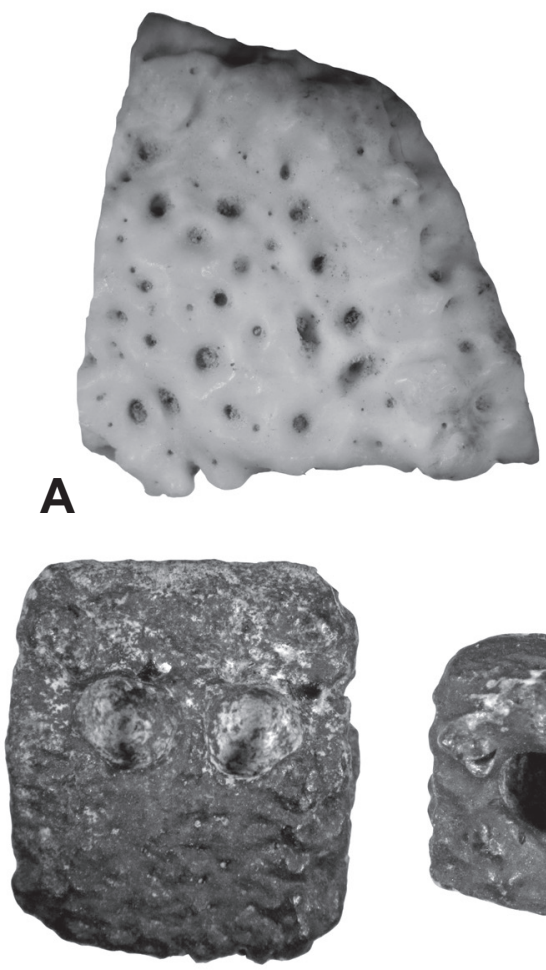

D

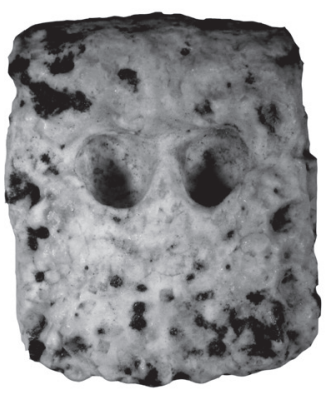

H

E

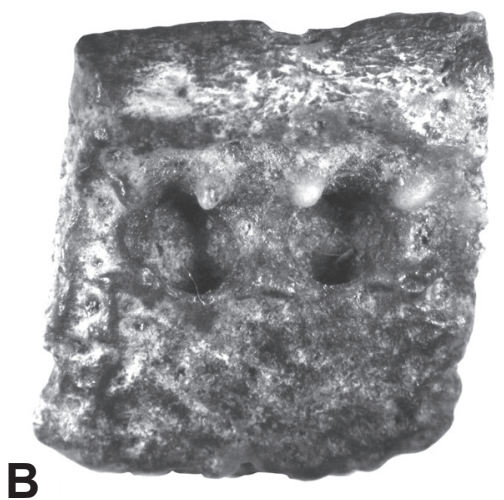

B

C
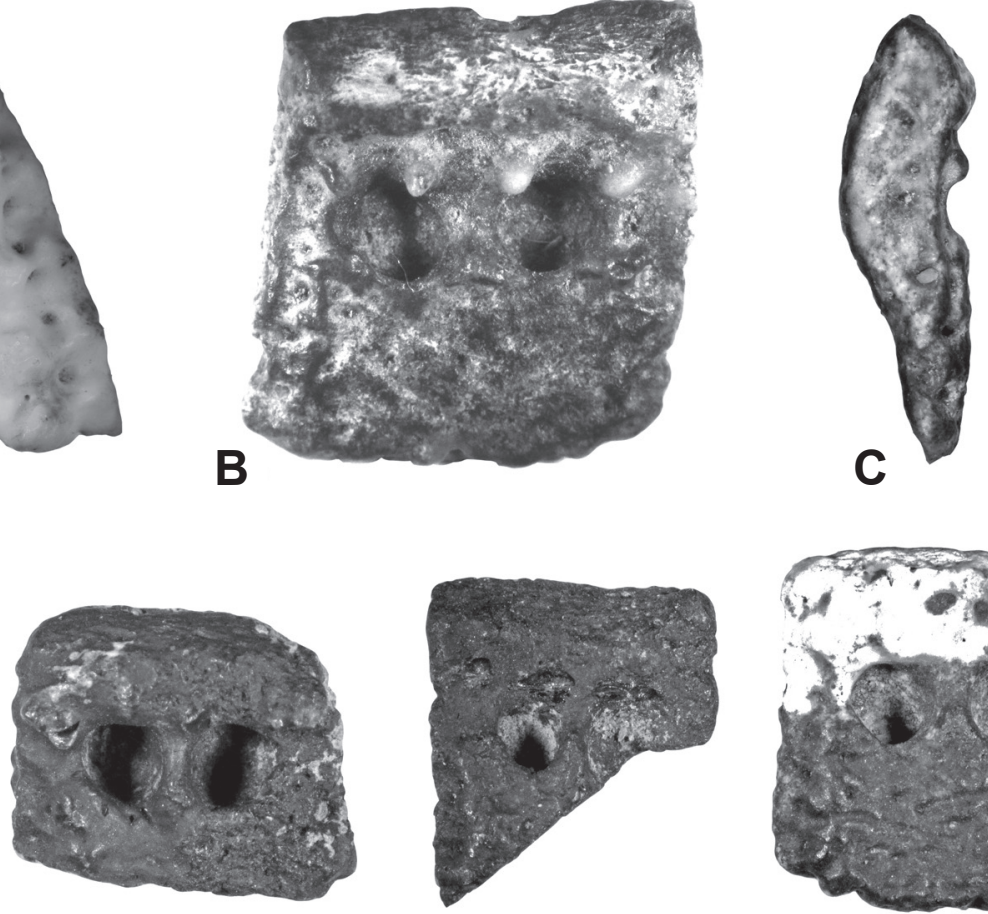

$\mathbf{F}$
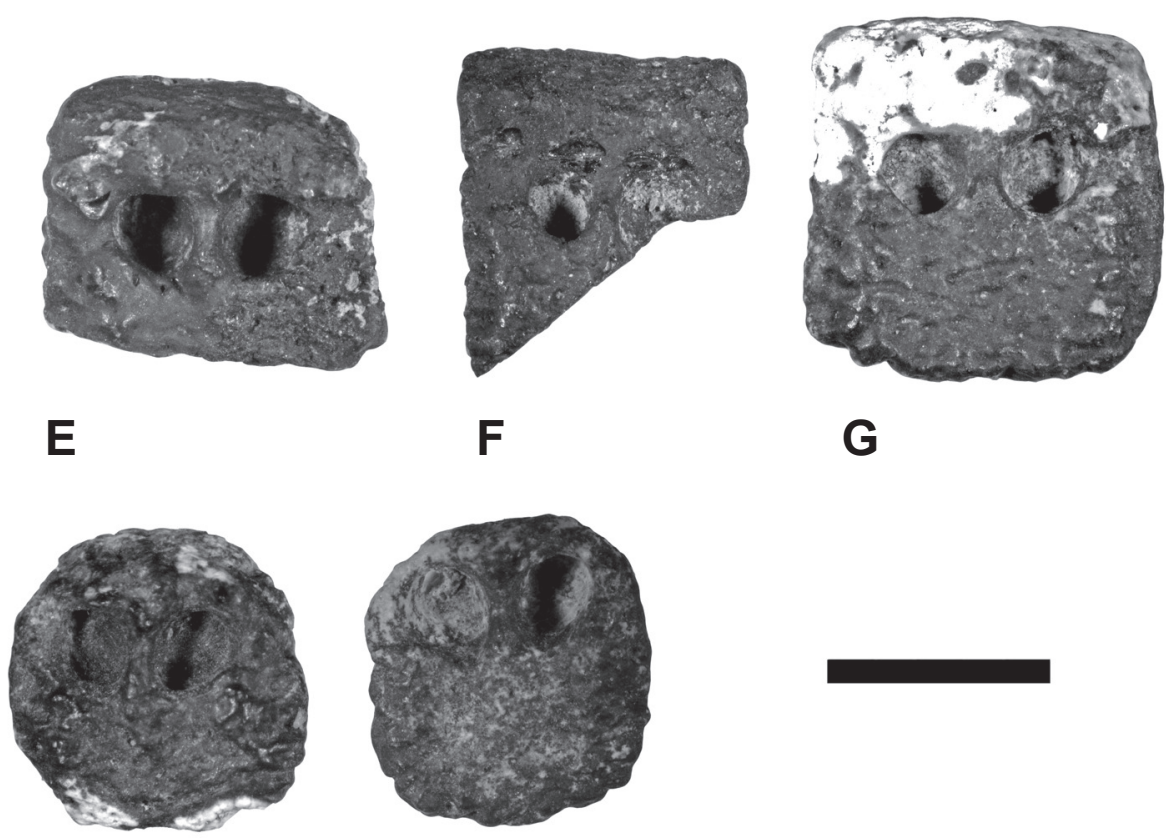

$\mathbf{J}$

Figura 3. Nuevos especímenes de Peltephilidae. A, Peltephilidae gen. et sp. indet., MLP 74-II-1-23, osteodermo cefálico en vista dorsal. B-C, Peltephilus pumilus, MLP 91-IV-1-62; B, osteodermo móvil en vistas dorsal; C, vista lateral; D-J, P. nanus, MLP 91-IV-1-35; D-G, osteodermos móviles en vista dorsal; MLP 91-IV-1-134; H, osteodermo móvil en vista dorsal; I-J, osteodermos fijos en vista dorsal. Escala = $5 \mathrm{~mm}$.

Figure 3. New specimens of Peltephilidae. A, Peltephilidae gen. et sp. indet., MLP 74-II-1-23, cephalic osteoderm in dorsal view; B-C, Peltephilus pumilus, MLP 91-IV-1-62; B, movable osteoderm in dorsal view; C, lateral view. D-J, P. nanus, MLP 91-IV-1-35; D-G, movable osteoderms in dorsal view; MLP 91-IV-1-134; H, movable osteoderm in dorsal view; I-J, fixed osteoderms in dorsal view. Scale bar $=5 \mathrm{~mm}$. 


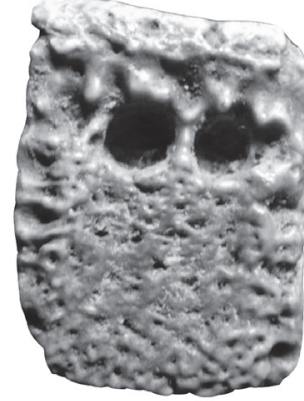

A

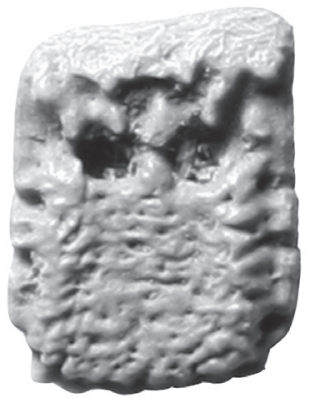

F

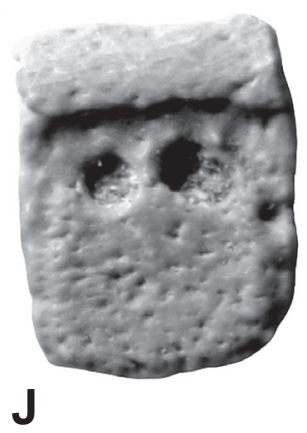

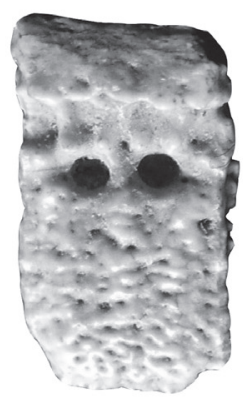

B

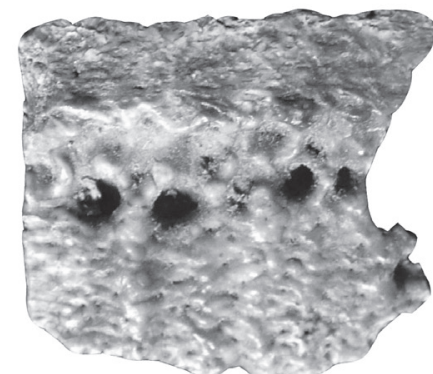

C

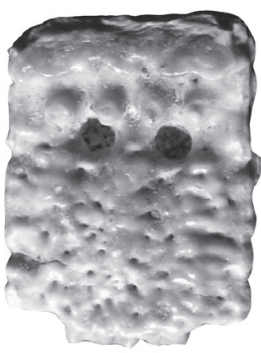

D

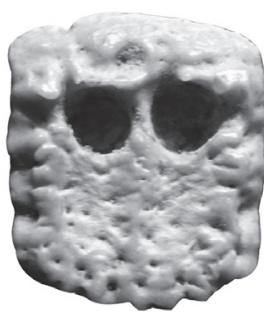

E

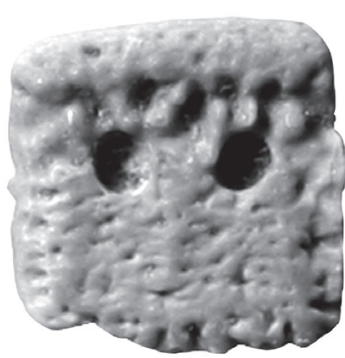

G

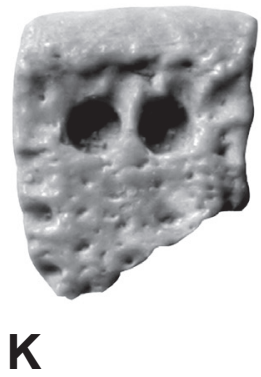

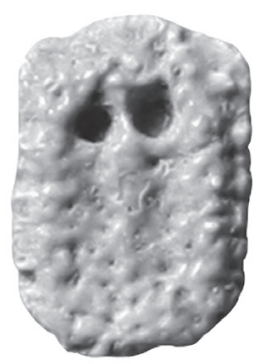

H

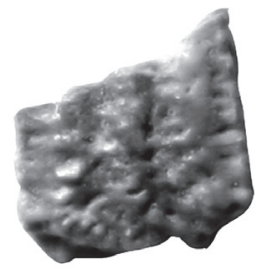

L

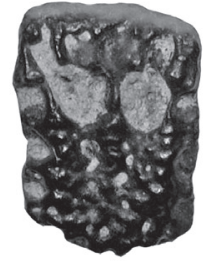

M

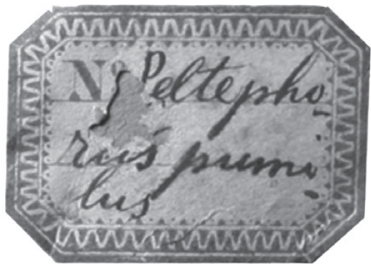

I

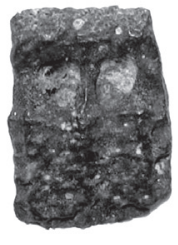

$\mathbf{N}$

Figura 4. Peltephilus pumilus. A-D, osteodermos móviles en vista dorsal; E, osteodermo fijo en vista dorsal (MACN A-866-870, ¿sintipo?, figurado por Ameghino, 1889: lám. LXIX, fig. 9). F-G, osteodermos móviles en vista dorsal; H, osteodermo fijo en vista dorsal, I, etiqueta original de F. Ameghino (MLP 67-XI-13-1a3, ¿sintipo?). J-L, osteodermos móviles en vista dorsal (MLP 67-VIII-12-1c, ¿sintipo?). M-N: P. nanus. M, osteodermo móvil en vista dorsal, figurado por Ameghino (1898: fig. 83.VII.b); N, osteodermo fijo en vista dorsal (MACNA-7958-7959, holotipo). Escala = 10 mm.

Figure 4. Peltephilus pumilus. A-D, movable osteoderms in dorsal view; E, fixed osteoderm in dorsal view (MACN A-866-870, ¿sintype? figured by Ameghino (1889: plate LXIX, fig. 9)). F-G, movable osteoderms in dorsal view; $\mathbf{H}$, fixed osteoderm in dorsal view; $\mathbf{I}$, original label of $F$. Ameghino (MLP 67-XI-13-1a3, sintype?). J-L, movable osteoderms in dorsal view (MLP 67-VIII-12-1c, sintype?). M-N: P. nanus. M, movable osteoderm in dorsal view, figured by Ameghino, 1898: fig. 83.VII.b); N, fixed osteoderm in dorsal view (MACN A-7958-7959, holotype). Scale bar = $10 \mathrm{~mm}$.

de P. nanus estudiados es mayor que en P. pumilus (ver Tabla 1).

Se describen tres nuevos osteodermos móviles completos que miden 8.2-9.2 de largo x 7.7-8.2 de ancho y los dos fijos 6.7-7.1 de largo x 6.3-6.7 de ancho. Si bien las diferencias de tamaño con los osteodermos de $P$. pumilus no son tan marcadas, son igualmente más chicos. A diferencia de P. pumilus no presenta los tubérculos en la región anterior ni las elevaciones longitudinales tan desarrolladas.

Finalmente, si bien Peltephilus nanus fue considerado nomen dubium por uno de los autores (González, 2010a,b) los nuevos especímenes aquí descriptos y las diferencias indicadas con $P$. pumilus justifican su validez.

\section{DISCUSIÓN}

Una de las modificaciones más notorias que propone la nueva nomenclatura para los Peltephilidae es el reemplazo del término figura central por el de elevación central longitudinal. Una figura es un área de la superficie externa del osteodermo delimitado por surcos (con o sin forámenes) (ver Fernicola \& Porpino, 2012 y Góis et al., 2013, para su uso en Glyptodontidae y Pampatheriidae).

La relación entre los elementos epidérmicos (escamas epidérmicas) y los osteodermos, dentro de los Dasypodidae es variable (Simpson, 1948). Los Dasypodinae (e.g. Dasypus Linnaeus, 1758; Propraopus Ameghino, 1881) tienen 
más de una escama epidérmica por osteodermo, la figura central (= figura principal o "main figure" sensu Oliveira \& Pereira, 2009; Porpino et al., 2010) posee su propia escama y las figuras laterales poseen escamas compartidas por dos, tres o hasta cuatro osteodermos (Holmes \& Simpson, 1931; Scillato-Yané, 1982; Wetzel \& Mondolfi, 1979; Wetzel, 1985; Vizcaíno, 1994; Carlini et al., 1997; Vickaryous \& Hall, 2006; Carlini et al., 2009). Algunos Euphractinae primitivos (i.e. Parutaetus chilensis Carlini, Ciancio, Flynn, Scillato-Yané \& Wyss, 2009) presentan una situación similar, con escamas que son compartidas por dos osteodermos adyacentes (Ciancio \& Carlini 2008b; Carlini et al., 2009, 2010; Ciancio, 2010). El resto de los Euphractinae pierde esta condición; dentro de los Euphractinae actuales, los Euphractini (Euphractus Wagler, 1830, Chaetophractus Fitzinger, 1871 y Zaedyus Ameghino, 1889) y los Chlamyphorini (Chlamyphorus Harlan, 1825), poseen una sola escama epidérmica por osteodermo (Yepes, 1928; Fernández, 1931; Simpson, 1948; Carlini et al., 2009; Krmpotic et al., 2009). Finalmente, dentro de los Tolypeutinae, los Tolypeutini (i.e. Tolypeutes Illiger, 1811) poseen una o más escamas por osteodermo (Lahille, 1895) y los Priodontini más de una aunque sin compartirlas con el osteodermo contiguo (e.g. Priodontes Cuvier, 1825) (Lahille, 1895; Yepes, 1928; Wetzel, 1985).

Los Chlamyphorini (Chlamyphorus) carecen de figuras delimitadas por surcos en la superficie externa del osteodermo aunque si poseen una elevación central longitudinal desarrollada ("figura central") (Lahille, 1895; Yepes, 1928; Scillato-Yané, 1982). La similitud entre los osteodermos de los Peltephilidae y los Chlamyphorini ya fue indicada por Lahille (1895) y entre los Peltephilidae y los Pampatheriidae por Ameghino $(1889,1902)$. Asimismo en las descripciones comparativas de los Peltephilidae, Ameghino $(1887,1889,1898)$ usualmente utilizaba al género Priodontes por lo que se puede interpretar que reconoció ciertas similitudes con los Priodontini también. En este sentido, los osteodermos de los Pampatheriidae estaban cubiertos por una única escama epidérmica ("keratinous surface" sensu Holmes \& Simpson, 1931; Edmund, 1987) y por analogía los mismo habría ocurrido con los Peltephilidae, dado que ninguno de los dos últimos presenta figuras desarrolladas, pero si, en ocasiones, elevaciones centrales longitudinales.

Teniendo en cuenta que se ha utilizado figura central o figura longitudinal media como sinónimo de cualquier elevación presente en la superficie externa del osteodermo de los Peltephilidae (Ameghino, 1887, 1889, 1897, 1920; Bordas, 1936; Scillato-Yané, 1982, 1977), y que dichas elevaciones no son estrictamente figuras dado que no están delimitadas por surcos y no reflejan la posición de escamas corneas como en la mayoría de los Dasypodidae, se propone describir la superficie esculpida externa de los osteodermos de Peltephilidae (al igual que la de los Pampatheriidae) en términos de un área o región central, delimitada por elevaciones marginales y ornamentada con depresiones y elevaciones longitudinales de diferentes morfologías (i.e. angostas, amplias, bajas, elevadas). El término elevación longitudinal fue utilizado una sola vez por Ameghino (1889) para describir osteodermos de
Peltephilus aunque desde entonces no ha sido utilizado en ese sentido; este término también reemplaza "ridge", "crest" y "keel" aplicado a los Peltephilidae (Scott, 1903; Croft et al., 2007, 2009; Kramarz et al., 2010; González Ruiz et al., 2012).

El número de especies de Peltephilidae disminuye progresivamente durante el Neógeno desde el Mioceno temprano tardío (Santacrucense) hasta desaparecer durante el Mioceno tardío (Chasiquense).

Para el Santacrucense se han descripto numerosas especies en Patagonia (Ameghino, 1887, 1891, 1894, 1898, 1906; Moreno \& Mercerat, 1891; Scott, 1903; Bordas, 1936; Scillato-Yané, 1980); además de dos registros fuera del ámbito patagónico en más bajas latitudes en la Provincia de San Juan (Argentina) y en Chucal (Chile) (Croft et al., 2007; López et al., 2011).

En cambio, para las otras faunas tradicionalmente incluidas en el Mioceno medio los registros son sumamente escasos. Se ha mencionado una probable nueva especie de Peltephilidae para el Friasense s.s. (Chile) (Bostelmann et al., 2012) y un Peltephilidae gen.? et sp. nov. para la fauna equivalente de Cerdas (Bolivia) (Croft et al., 2009). Para el Colloncurense, se ha citado la presencia de Peltephilidae en Patagonia, pero como mencionado anteriormente, no se han encontrado los materiales correspondientes a estas referencias previas a este manuscrito. Hay un registro de cf. Peltephilus sp. para la probable fauna colloncurense de Nazareno (Bolivia) (Oiso, 1991), sin embargo su asignación al Colloncurense no ha sido corroborada (Croft et al., 2009). En la parte final del Mioceno medio, en el Mayoense (Argentina) se registra Epipeltephilus recurvus Ameghino, 1904 (Ameghino, 1904; Rovereto, 1914; Roth, 1920; Kraglievich, 1930; Scillato-Yané \& Carlini, 1998).

Por último, el registro de Epipeltephilus kanti González Ruiz, Scillato-Yané, Krmpotic \& Carlini, 2012 durante el Chasiquense (Argentina) indica la extinción de la familia en el Mioceno tardío (González Ruiz et al., 2012).

Peltephilus pumilus se ha registrado hasta ahora en la fauna Pinturense de Gran Barranca (Formación Sarmiento, Mioceno temprano, 19.7-18.7 Ma.) (Kramarz et al., 2010) y en el Santacrucense (Formación Santa Cruz, Mioceno temprano tardío, 16.5 Ma.) (Ameghino, 1887, 1900-1902), mientras que P. nanus se ha registrado exclusivamente en Formación Santa Cruz (Santacrucense, Mioceno temprano tardío) (Ameghino, 1898, 1900-1902). Los nuevos registros de P. pumilus y P. nanus aquí presentados para el Colloncurense (Formación Collón Curá, Mioceno medio, 15.7 Ma.) extienden el biocrón de ambas especies en $c a$. 1 Ma. y aportan evidencia a favor de la mayor similitud faunística entre el Colloncurense y Santacrucense, que entre el Colloncurense y el MayoenseChasiquense, en congruencia con otros estudios (Bond, 1993; Vucetich et al., 1993; Ortiz Jaureguizar et al., 1993; González Ruiz et al., 2012).

\section{CONCLUSIONES}

Se describe por primera vez Peltephilidae para el Colloncurense (Mioceno medio) y se identifican, al menos, las especies Peltephilus pumilus y P. nanus. 
Peltephilus pumilus posee en promedio osteodermos con forámenes de menor tamaño en relación al área total del osteodermo que $P$. nanus.

La presencia de Peltephilus pumilus y P. nanus en el Colloncurense sugieren una mayor afinidad del Colloncurense con el Santacrucense que con el Laventense, Mayoense o Chasiquense.

Se propone utilizar "elevación central longitudinal" en lugar de "figura central" en la descripción de los osteodermos de los Peltephilidae, dado que en estos la elevación citada no representa la posición de una escama epidérmica suprayacente como en los Dasypodidae que si poseen figuras delimitadas por surcos.

Por analogía con los osteodermos de Dasypodidae actuales (Chlamyphorini) y con los Pampatheriidae, cada osteodermo de la coraza de los Peltephilidae habría estado cubierto por una única escama epidérmica.

\section{AGRADECIMIENTOS}

Los autores desean agradecer a los encargados de las colecciones M. Reguero (MLP), S. Alvarez (MACN), A. Kramarz (MACN) y J. Fernicola (MACN) que permitieron el acceso a los especímenes estudiados. A G. Martin y G. Montoya por fotografías del espécimen MACN A 866-870 y MACN A 7958-7959, respectivamente. A U. Pardiñas (CENPAT) por información acerca de los especímenes de Peltephilidae dados a conocer y por sugerencias acerca del manuscrito. A la editora A. Ribeiro (RBP) por su buena predisposición durante la edición de esta contribución. A los revisores C. Krmpotic (MLP) y E. Olivera (UFPE) por sus valiosos comentarios que mejoraron este trabajo.

\section{REFERENCIAS}

Abrantes, E.A.L. \& Bergqvist, L.P. 2006. Proposta filogenética para os Dasypodidae (Mammalia: Cingulata). In: V. Gallo; P.M. Brito; H.M.A Silva \& F.J. Figueiredo (eds.) Paleontología de Vertebrados: Grandes Temas e Contribuições Científicas, Interciência, 1:216-274.

Ameghino, F. 1887. Enumeración sistemática de las especies de mamíferos fósiles coleccionados por Carlos Ameghino en los terrenos eocenos de Patagonia austral y depositados en el Museo La Plata. Boletín del Museo La Plata, 1:1-26.

Ameghino, F. 1889. Contribución al conocimiento de los mamíferos fósiles de la República Argentina. Actas de la Academia Nacional de Ciencias en Córdoba, 6:1-1027.

Ameghino, F. 1891. Nuevos restos de mamíferos fósiles descubiertos por Carlos Ameghino en el Eoceno inferior de la Patagonia austral. Especies nuevas, adiciones y correcciones. Revista Argentina de Historia Natural, 1:289-328.

Ameghino, F. 1894. Enumération synoptique des espèces de mammifères fossiles des formations éocènes de Patagonie. Boletín de la Academia Nacional de Ciencias en Córdoba, 13:259-455.

Ameghino, F. 1895. Sur les édentés fossiles de l'Argentine. Examen critique, révision et correction de l'ouvrage de M. R. Lydekker "The extinct edentates of Argentina". Revista del Jardin Zoológico de Buenos Aires, 3:97-192. Obra incompleta ver Ameghino, F. 1920.
Ameghino, F. 1897. Mammifères crétacés de l'Argentine. Boletín del Instituto Geográfico Argentino, 18:406-429,431-521.

Ameghino, F. 1898. Sinópsis Geológico-Paleontológica. Segundo Censo de la República Argentina, 1:111-255.

Ameghino, F. 1900-1902. L'age des formations sédimentaires de Patagonie. Anales de la Sociedad Cientifica Argentina, 50:109130,145-165, 207-229 (1900); 51:20-39,65-91(1901); 52:189197,244-250 (1901); 54:161-180,220-240,283-342 (1902).

Ameghino, F. 1902. Première contribution à la connaissance de la faune mammalogique des couches à Colpodon. Boletín de la Academia Nacional de Ciencias en Córdoba, 17:71-138.

Ameghino, F. 1904. Nuevas especies de mamíferos cretáceos y terciarios de la República Argentina. Anales de la Sociedad Cientifica Argentina, 58:35-41,56-71,182-192,225-291.

Ameghino, F. 1906. Les formations sédimentaires du Crétacé superieur et du Tertiaire de Patagonie, avec un parallèle entre leurs faunes mammalogiques et celles de l'ancient continent. Anales del Museo Nacional de Historia Natural de Buenos Aires, 3:1-568.

Ameghino, F. 1920. Sur les édentés fossiles de l'Argentine. Examen critique, révision et correction de l' ouvrage de M. R. Lydekker "The exinct edentates of Argentina", etc. (Ouvrage à démi-inédite avec des notes additionelles sur quelques ongulés et carnassiers). In: A.J. Torcelli (ed.) Obras Completas y Correspondencia Cientifica de Florentino Ameghino, Taller de Impresiones Oficiales del Gobierno de la Provincia de Buenos Aires, 11:447-909.

Billet, G.; Hautier, L.; de Muizon, C. \& Valentin, X. 2011. Oldest cingulate skulls provide congruence between morphological and molecular scenarios of armadillo evolution. Proceedings of the Royal Society, 278:2791-2797. doi: 10.1098/rspb.2010.2443

Bond, M. 1993. Comentarios sobre los Ungulados de la Edad Friasense de Patagonia, Argentina. Ameghiniana, 30:350R.

Bondesio, P.; Rabassa, J.; Pascual, R.; Vucetich, M.G. \& Scillato Yané, G.J. 1980. La Formación Collón-Curá de Pilcaniyeu viejo y sus alrededores (Río Negro, República Argentina). Su antigüedad y las condiciones ambientales según su distribución de litogénesis y sus vertebrados: In: CONGRESO ARGENTINO DE PALEONTOLOGÍA Y BIOESTRATIGRAFÍA Y CONGRESO LATINOAMERICANO DE PALEONTOLOGÍA, 2-1, 1978. Actas, Buenos Aires, p. 85-99.

Bordas, A.F. 1936. Los Peltateloidea de la colección Ameghino. Peltephiloda. (Infraorden de los Xenarthra). Physis, 12:1-18.

Bostelmann, E.; Bobe, R.; Carrasco, G.; Alloway, B.; Santi-Malnis P.; Mancuso, A.; Agüero, B.; Alemseged, Z. \& Godoy, Y. 2012. The Alto Río Cisnes Fossil Fauna (Río Frías Formation, Early Middle Miocene, Friasian SALMA): a keystone and paradigmatic vertebrate assemblage of the South American Fossil Record. In: SIMPOSIO-PALEONTOLOGÍA EN CHILE, 3, 2012. Actas, Punta Arenas, p. 42-45.

Carlini, A.A.; Vizcaíno, S.F. \& Scillato-Yané, G.J. 1997. Armored Xenarthrans: a unique taxonomic and ecologic assemblage. In: F.R. Kay; R.H. Madden; R.L. Cifelli \& J.J. Flynn (eds.) Vertebrate Paleontology in the Neotropics. The Miocene Fauna of La Venta, Colombia, Smithsonian Institution Press, p. 213-226.

Carlini, A.A.; Ciancio, M.R.; Flynn, J.J.; Scillato-Yané, G.J. \& Wyss, A.R. 2009. The phylogenetic and biostratigraphic significance of new armadillos (Mammalia, Xenarthra, Dasypodidae, Euphractinae) from the Tinguirirican (Early Oligocene) of Chile. Journal of Systematic Palaeontology, 7:489-503. doi: 10.1017/ S1477201908002708 
Carlini, A.A.; Ciancio, M.R. \& Scillato-Yané, G.J. 2010. Middle Eocene - Early Miocene Dasypodidae (Xenarthra) of southern South America: faunal succesion at Gran Barranca - biostratigraphy and paleoecology. In: R.H. Madden; A.A. Carlini; M.G. Vucetich \& R.F. Kay (eds.) The Paleontology of Gran Barranca. Environmental Change through the Middle Cenozoic of Patagonia, Cambridge University Press, p. 106-129.

Casamiquela, R.M. 1963. Sobre un par de anuros del Mioceno de Río Negro (Patagonia) Wawelia gerholdi n. gen. et sp. (Ceratophrydidae) y Gigantobatrachus parodii (Leptodactylidae). Ameghiniana, 3:141-160.

Cazau, L.B.; Mancini, J.; Cangini, J. \& Spalleti, L. 1989. Cuenca de Ñirihuau. In: G.A. Chebli \& L.A. Spalletti (eds.) Cuencas Sedimentarias Argentinas. Instituto Superior de Correlación Geológica, p. 299-318. (Serie Correlación Geológica 6).

Ciancio, M.R. 2010. Los Dasypodoidea (Mammalia, Xenarthra) del Deseadense (Oligoceno) de América del Sur. Su importancia filogenética y bioestratigráfica. Facultad de Ciencias Naturales y Museo, Universidad Nacional de La Plata, Tesis Doctoral, 290 p.

Ciancio, M.R. \& Carlini, A.A. 2008a. Identificación de ejemplares tipo de Dasypodidae (Mammalia, Xenarthra) del Paleógeno de Argentina. Revista del Museo Argentino de Ciencias Naturales "Bernardino Rivadavia", 10:221-237.

Ciancio, M.R. \& Carlini, A.A. 2008b. Cambios en el patrón morfológico de los osteodermos de Dasypodidae (Xenarthra, Mammalia). In: CONGRESO LATINOAMERICANO DE PALEONTOLOGÍA DE VERTEBRADOS, 3, 2008. Resúmenes, Neuquén, p. 58.

Croft, D.A.; Anaya, F.; Auerbach, D.; Garzione, C. \& MacFadden, B.J. 2009. New Data on Miocene Neotropical Provinciality from Cerdas, Bolivia. Journal of Mammal Evolution, 16:175-198. doi: 10.1007/s10914-009-9115-0

Croft, D.A.; Flynn, J.J. \& Wyss, A.R. 2007. A new basal Glyptodontoid and other Xenarthra of the early Miocene Chucal Fauna, Northern Chile. Journal of Vertebrate Paleontology, 27:781-797. doi: 10.1671/0272-4634(2007)27[781:ANBGAO ]2.0.CO;2

Coira, B.L. 1979. Descripción Geológica de la Hoja 40d, Ingeniero Jacobacci, Provincia de Río Negro. Servicio Geológico Nacional, 94 p. (Boletín 168).

Cope, E.D. 1889. The Edentata of North America. The American Naturalist, 23:657-664.

Cooper, Z.K. 1930. A histological study of the integument of the armadillo, Tatusia novemcincta. The American Journal of Anatomy, 45:1-37.

Cucchi, R.; Espejo, P. \& González, R. 1998. Hoja Geológica 4169-I Piedra del Águila Provincias del Neuquén y Río Negro. Servicio Geológico Minero Argentino, 74 p. (Boletín 242).

de Barrio, R.E.; Scillato-Yané, G.J. \& Bond, M. 1984. La Formación Santa Cruz en el borde occidental del macizo del Deseado (Provincia de Santa Cruz) y su contenido paleontológico: In: CONGRESO GEOLÓGICO ARGENTINO, 9, 1984. Actas, S.C. de Bariloche, p. 539-556.

Edmund, G.A. 1985. The Armor of fossil giant armadillos (Pampatheriidae, Xenarthra, Mammalia). Texas Memorial Museum, 20 p. (Pearce-Sellards-Series 40).

Edmund, G.A. 1987. Evolution of the genus Holmensia (Pampatheriidae, Mammalia) in Florida, with remarks on taxonomy and distribution. Texas Memorial Museum, $20 \mathrm{p}$. (Pearce-Sellards Series 45).

Engelmann, G.F. 1985. The Phylogeny of the Xenarthra. In: G.G. Montgomery (ed.) The Evolution and Ecology of Armadillos, Sloths, and Vermilinguas, Smithsonian Institution Press, p. 51-64.
Fernández, M. 1931. Sobre la anatomía microscópica y embriología de la coraza de Dasypus villosus Desm. Actas de la Academia Nacional de Ciencias, 10:61-121.

Fernicola, J.C. \& Porpino, K.O. 2012. Exoskeleton and Systematics: a Historical Problem in the Classification of Glyptodonts. Journal of Mammal Evolution, 19:171-183. doi: 10.1007/ s10914-012-9186-1

Flynn, J.J. \& Swisher III C.C. 1995. Cenozoic South American Land Mammal Ages: Correlation to Global Geochronologies. Geochronology Time Scales and Global Stratigraphic Correlation, 317-333 p. (SEMP Special Publication 54).

Galli, C.A. 1969. Descripción Geológica de la Hoja 38c, Piedra del Aguila, Provincias de Neuquén y Río Negro. Dirección Nacional de Geología y Minería, 67 p. (Boletín 111).

Ganduglia, P.R. 1977. Observaciones geológicas en la región de Ingeniero Jacobacci provincia de Río Negro (con énfasis en el Cretácico y Terciario). Facultad de Ciencias Naturales, Universidad Nacional de Buenos Aires, Tesis de licenciatura, $86 \mathrm{p}$.

Gaudin, T.J. \& Wible, J.R. 2006. The Phylogeny of Living and Extinct Armadillos (Mammalia, Xenarthra, Cingulata): Craniodental Analysis. In: M.T. Carrano; R.J. Gaudin; W.R. Blob \& J.R. Wible (eds.) Amniote Paleobiology. Perspectives on the Evolution of Mammals, Birds, and Reptiles, The University of Chicago Press, p. 153-198.

Góis, F.; Scillato-Yané, G.J.; Carlini, A.A. \& Guilherme, E. 2013. A new species of Scirrotherium Edmund \& Theodor, 1997 (Xenarthra, Cingulata, Pampatheriidae) from the late Miocene of South America. Alcheringa, 37:175-186 doi: 10.1080/03115518.2013.733510

González, L.R. 2010a. Los Cingulata (Mammalia, Xenarthra) del Mioceno temprano y medio de Patagonia (edades Santacrucense y “Friasense"). Revisión sistemática y consideraciones bioestratigráficas. Facultad de Ciencias Naturales y Museo, Universidad Nacional de La Plata, Tesis doctoral, 471 p.

González, L.R. 2010b. Los Cingulata (Mammalia, Xenarthra) del Mioceno temprano y medio de Patagonia (edades Santacrucense y "Friasense"). Revisión sistemática y consideraciones bioestratigráficas. Mastozoología Neotropical (Resúmenes de Tesis), 17:402-403.

González, L.R. \& Scillato-Yané, G.J. 2008. Una nueva especie de Stegotherium (Xenarthra, Dasypodidae, Stegotheriini) del Mioceno de la provincia de Santa Cruz. Ameghiniana, 45:641-648.

González-Ruiz, L.R.; Scillato-Yané, G.J.; Krmpotic, C.M. \& Carlini, A.A. 2012. A new species of Peltephilidae (Mammalia: Xenarthra: Cingulata) from the late Miocene (Chasicoan SALMA) of Argentina. Zootaxa, 3359:55-64.

Grassé, P.P. 1955. Ordre des Édentés. In: P.P. Grassé (ed.) Traité de Zoologie. Anatomie, Systématique, Biologie, Masson et Cie Editeurs, p. 1182-1266.

Gray, J.E. 1821. Catalogue of Carnivorous, Pachydermatous, and Edentate Mammalia in the British Museum. London, British Museum, $398 \mathrm{p}$.

Hill, R.V. 2006. Comparative anatomy and histology of xenarthran osteoderms. Journal of Morphology, 267:1441-1460. doi: 10.1002/jmor. 10490

Hoffstetter, R. 1958. Xenarthra. In: J. Piveteau (ed.) Traité de Paléontologie. Masson et Cie Editeurs, p. 535-647.

Holmes, W.W. \& Simpson, G.G. 1931. Pleistocene exploration and fossil Edentates in Florida. Bulletin of the American Museum of Natural History, 59:383-418. 
Illiger, C. 1811. Prodromus systematis mammalium et avium additis terminis zoographicis uttriusque classis. Berlin, C. Salfeld, 301 p.

Kraglievich, L. 1930. La Formación Friaseana del río Frias, río Fénix, Laguna Blanca, etc., y su fauna de mamíferos. Physis, 10:127-161.

Kramarz, A.G.; Vucetich, M.G.; Carlini, A.A.; Ciancio, M.R.; Abello, M.A.; Deschamps, C.M. \& Gelfo, J.N. 2010. A new mammal fauna at the top of the Gran Barranca sequence and its biochronological significance. In: R.H. Madden; A.A. Carlini; M.G. Vucetich \& R.F. Kay (eds.) The Paleontology of Gran Barranca. Evolution and Environmental Change through the Middle Cenozoic of Patagonia, Cambridge University Press, p. 264-277.

Krmpotic, C.M.; Ciancio, M.R.; Barbeito, C.; Mario, R.C. \& Carlini, A.A. 2009. Osteoderm morphology in recent and fossil euphractine xenarthrans. Acta Zoológica, 90:339-351. doi: 10.1111/j.1463-6395.2008.00359.x

Lahille, F. 1895. Contributions a l'étude des Édentés a bandes mobiles de la République Argentine. Anales del Museo de La Plata, 2:1-32.

Leanza, H.A.; Herrero, J.C.; Donnari, E. \& Pucci, J.C. 1997. Hoja Geológica 3969-III Picún Leufú Provincias del Neuquén y Río Negro. Servicio Geológico Minero Argentino, 121 p. (Boletín 218).

López, G. M.; Vucetich, G.M.; Carlini, A.A.; Bond, M.; Pérez, M.E.; Ciancio, M.R.; Pérez, D.J.; Arnal, M. \& Olivares, A.I. 2011 New Miocene mammal assemblages from Neogene Manantiales basin, Cordillera Frontal, San Juan, Argentina. In: J.A. Salfity \& R.A. Marquillas (eds.) Cenozoic Geology of the Central Andes of Argentina, SCS Publisher, p. 211-226.

Lydekker, R. 1894. Contribution to a knowledge of the fossil Vertebrates of Argentina. 2. The extinct Edentates of Argentina. Anales del Museo de La Plata, 3:1-118.

Madden, R.H.; Guerrero J.; Kay R.F.; Flynn, J.J.; Swisher III, C.C. \& Walton, A.H. 1997. The Laventan Stage and Age. Vertebrate Paleontology in the Neotropics. In: F.R. Kay; R.H. Madden; R.L. Cifelli \& J.J. Flynn (eds.) Vertebrate Paleontology in the Neotropics. The Miocene Fauna of La Venta, Colombia, Smithsonian Institution Press, p. 499-519.

Marshall, L.G.; Pascual, R.; Curtis, G. H. \& Drake, R.E. 1977. South American Geochronology: Radiometric Time Scale for Middle to Late Tertiary Mammal-Bearing Horizons in Patagonia. Science, 195:1325-1328. doi: 10.1126/science.195.4284.1325

Mazzoni, M.M. 1993. Sedimentación Colloncurense en las provincias de Río Negro y Neuquén. Ameghiniana, 30:351.

Mazzoni, M.M. \& Benvenuto, A. 1990. Radiometric ages of tertiary ignimbrites and the Collon Cura Formation, northwestern Patagonia. In: CONGRESO GEOLÓGICO ARGENTINO, 11, 1990. Actas, San Juan, p. 87-90.

Mazzoni, M.M. \& Stura, S. 1990. El Miembro Ignimbrítico Pilcaniyeu, Formación Collón-Curá (Mioceno), provincias de Río Negro y Neuquén. In: REUNIÓN ARGENTINA DE SEDIMENTOLOGÍA, 3, 1990. Actas, San Juan, p. 187-192.

McKenna, M.C. \& Bell, S.K. 1997. Classification of Mammals above the Species Level. New York, Columbia University Press, 640 p.

Moreno, F.P. \& Mercerat, A. 1891. Notas sobre algunas especies de un género aberrante de los Dasypoda (eoceno de Patagonia) conservadas en el Museo de La Plata. Revista del Museo de La Plata, 2:57-63.

Nullo, F.E. 1978. Descripción geológica de la Hoja 41d, Lipetrén, Provincia de Río Negro. Servicio Geológico Nacional, 88 p. (Boletín 158).
Oiso, Y. 1991. New Land Mammal Locality of Middle Miocene (Colloncuran) Age from Nazareno, Southern Bolivia. Revista Técnica de YPFB, 12:653-672.

Oliveira, E.V. \& Bergqvist, L.P. 1998. A new Paleocene Armadillo (Mammalia, Dasypodoidea) from the Itaborai Basin, Borazil. Asociación Paleontológica Argentina, p. 35-40 (Publicación Especial 5).

Oliveira, E.V. \& Pereira, J.C. 2009. Intertropical cingulates (Mammalia, Xenarthra) from the Quaternary of southern Brazil: systematics and paleobiogeographical aspects. Revista Brasileira de Paleontologia, 12:167-178. doi:10.4072/ rbp.2009.3.01

Ortiz Jaureguizar, E.; Madden, R.; Vucetich, G.; Bond, M.; Carlini, A.; Goin, F.; Scillato-Yané, G. \& Vizcaíno, S. 1993. Un análisis de similitud entre las faunas de la "Edad-Mamífero Friasense". Ameghiniana, 30:351-352R.

Pascual, R. \& Odreman Rivas, O. 1973. Las unidades estratigráfias del terciario portadoras de mamíferos. Su distribución y sus relaciones con los acontecimientos diastróficos. In: CONGRESO GEOlÓGICO ARGENTINO, 5, 1973. Actas, Carlos Paz, p. 293-338.

Pascual, R., Vucetich, M.G. \& Scillato-Yané, G. J. 1990. Extinct and Recent South American and Caribbean Megalonychidae Edentates and Hystrycognathi rodents: outstanding examples of isolation. In: INTERNATIONAL SYMPOSIUM ON BIOGEOGRAPHICALASPECTS OF INSULARITY, 85, 1987. Atti dei Convegni Lincei, Rome, p. 627-640.

Pascual, R.; Bondesio, P.; Vucetich, M.G.; Scillato-Yané, G.J.; Bond, M. \& Tonni, E.P. 1984. Vertebrados fósiles Cenozoicos. In: CONGRESO GEOLÓGICO ARGENTINO, 9, 1984. Relatorio, S.C. de Bariloche, p. 439-461.

Pardiñas, U.F.J. 1991. Primer registro de primates y otros vertebrados para la Formación Collón Curá (Mioceno medio) del Neuquén, Argentina. Ameghiniana, 28:197-199.

Pardiñas, U.F.J. \& Reguero, M.A. 1987. Vertebrados fósiles de Cerro Castillo (Departamento Pilcaniyeu). Nueva localidad para el neógeno de Río Negro. In: JORNADAS ARGENTINAS DE PALEONTOLOGÍA DE VERTEBRADOS, 4, 1987. Resúmenes, Comodoro Rivadavia, p. 28-30.

Perea, D. 2005. Pseudoplohohorus absolutus n. sp. (Xenarthra, Glyptodontidae), variabilidad en Sclerocalyptinae y redefinición de una biozona del Mioceno Superior de Uruguay. Ameghiniana 42:175-190.

Porpino, K.O.; Fernicola, J.C. \& Bergqvist, L.P. 2010. Revisiting the intertropical Brazilian species Hoplophorus euphractus (Cingulata, Glyptodontoidea) and the phylogenetic affinities of Hoplophorus. Journal of Vertebrate Paleontology, 30:911-927. doi: 10.1080/02724631003765735

Rabassa, J. 1975. Geología de la región de Pilcaniyeu-Comallo (Provincia de Río Negro, Argentina). Fundación Bariloche, 128 p. (Publicación 17).

Rabassa, J. 1978. Estratigrafía de la región Pilcaniyeu. Comallo, Provincia de Río Negro. In: CONGRESO GEOLÓGICO ARGENTINO, 7, 1978. Actas, Neuquén, p. 731-746.

Roth, S. 1899. Apuntes sobre la geología y la paleontología de los territorios del Río Negro y Neuquén. Revista del Museo de La Plata, 9:141-172.

Roth, S. 1920. Investigaciones geológicas en la Llanura Pampeana. Revista del Museo de La Plata, 25:135-342.

Rovereto, C. 1914. Los estratos araucanos y sus fósiles. Anales del Museo Nacional de Historia Natural de Buenos Aires, 25:1-247. 
Scillato-Yané, G.J. 1977. Notas sobre los Dasypodidae (Mammalia, Edentata) del Plioceno del territorio Argentino. I. Los restos de Edad Chasiquense (Plioceno inferior) del sur de la provincia de Buenos Aires. Ameghiniana, 14:133-144.

Scillato-Yané, G.J. 1980. Catálogo de los Dasypodidae fósiles (Mammalia, Edentata) de la República Argentina. In: CONGRESO ARGENTINO DE PALEONTOLOGÍA Y BIOESTRATIGRAFÍA/CONGRESO LATINOAMERICANO DE PALEONTOLOGÍA, 2/1, 1978. Actas, Buenos Aires, p. 7-36.

Scillato-Yané, G.J. 1982. Los Dasypodidae (Mammalia, Edentata) del Plioceno y Pleistoceno de Argentina. Facultad de Ciencias Naturales y Museo, Universidad Nacional de La Plata, 159 p.

Scillato-Yané, G.J. 1986. Los Xenarthra fósiles de Argentina (Mammalia, Edentata). In: CONGRESO ARGENTINO DE PALEONTOLOGÍA Y BIOESTRATIGRAFÍA, 4, 1986. Actas, Mendoza, p. 151-155.

Scillato-Yané, G.J. \& Carlini, A.A. 1998. Nuevos Xenarthra del Friasense (Mioceno medio) de Argentina. Studia Geologica Salmanticensia, 34:43-67.

Scott, W.B. 1903. Mammalia of the Santa Cruz. Volume V, Paleontology. Part I, Edentata. 1. Dasypoda. In: W.B. Scott (ed.) Reports of the Princeton University Expeditions to Patagonia 1896-1899, Princeton University Press, p. 1-106.

Simpson, G.G. 1948. The beginning of the Age of Mammals in South America. Bulletin of the American Museum of Natural History, 91:1-232. doi: 10.1111/j.1469-185X.1937.tb01220.x

Tejedor, M.F.; Goin, F.J.; Gelfo, J.N.; López, G.; Bond, M.; Carlini, A.A.; Scillato-Yané, G.J.; Woodburne, M.O.; Chornogubsky, L.; Aragón, E.; Rueguero, M.A.; Czaplewski, N.J.; Vincon, S.; Martin, G.M. \& Ciancio, M.R. 2009. New Early Eocene Mammalian Fauna from Western Patagonia, Argentina. American Museum Novitates, 3638:1-43. doi: 10.1206/577.1.
Vickaryous, M.K. \& Hall, B.K. 2006. Osteoderm Morphology and Development in the Nine-Banded Armadillo, Dasypus novemcinctus (Mammalia, Xenarthra, Cingulata). Journal of Morphology, 267:1273-1283. doi: 10.1002/jmor.10475

Vizcaíno, S.F. 1994. Sistemática y Anatomía de los Astegotheriini Ameghino, 1906 (nuevo rango) (Xenarthra, Dasypodidae, Dasypodinae). Ameghiniana, 31:3-13.

Vizcaíno, S.F.; Rinderknecht, A. \& Czerwonogora, A. 2003. An enigmatic Cingulata (Mammalia: Xenarthra) from the late Miocene of Uruguay. Journal of Vertebrate Paleontology, 23:981-983. doi: 10.1671/2259-26

Vucetich, M. G.; Massoni, M.M. \& Pardiñas, U.F. 1993. Los roedores de la Formación Collón Curá (Mioceno medio) y la Ignimbrita Pilcaniyeu, Cañadón del Tordillo, Neuquén. Ameghiniana, 30:361-381.

Wetzel, R.M. 1985. The Identification and Distribution of Recent Xenarthra (=Edentata). In: Montgomery, G.G. (ed.) The Evolution and Ecology of Armadillos, Sloths, and Vermilinguas, Smithsonian Institution Press, p. 5-21.

Wetzel, R.M. \& Mondolfi, E. 1979. The Subgenera and Species of Long-nosed Armadillos, Genus Dasypus L. In: J.F. Eisenberg (ed.) Vertebrate Ecology in the Northern Neotropics, Smithsoniana Institution Press, p. 43-63.

Wolf, D. 2007. Osteoderm histology of extinct and recent Cingulata and Phyllophaga (Xenarthra, Mammalia): Implications for systematics and biomechanical adaptation. Hallesches Jahrbuch für Geowissenschaften, 23:145-151.

Yepes, J. 1928. Los «Edentata» Argentinos. Sistemática y Distribución. Revista de la Universidad de Buenos Aires, 2:1-55.

Received in November, 2012; accepted in June, 2013. 